\title{
Structures of Not-finitely Graded Lie Superalgebras
}

\section{Juanjuan Li and Guangzhe Fan*}

Department of Mathematics, Tongji University, Shanghai 200092, PR China

\begin{abstract}
This paper is devoted to investigating the structure theory of a class of not-finitely graded Lie superalgebras related to generalized super-Virasoro algebras. In particular, we completely determine the derivation algebras, the automorphism groups and the second cohomology groups of these Lie superalgebras.
\end{abstract}

Keywords: Not-finitely graded Lie superalgebras; Super-Virasoro algebras; Generalized super-Virasoro algebras; Derivations; Automorphisms; 2-Cocycles

\section{Introduction}

For the readers' convenience, we give some notations used in this paper. Let $\mathbb{Z} \subseteq \Gamma$ be an additive subgroup of $\mathbb{C}$ and $s \in \mathbb{C}$ such that $2 s \Gamma$. For simplicity, let $\Omega$ be an additive subgroup of $\mathbb{C}$ generated by $\Gamma \cup\{s\}$. Denote by $\mathbb{C}, \mathbb{C}^{*}, \mathbb{Z}, \mathbb{Z}_{+}, \Gamma^{*}, \Omega^{*}$ the sets of complex numbers, nonzero complex numbers, integers, nonnegative integers, nonzero elements of $\Gamma$, nonzero elements of $\Omega$, respectively. We assume that all vector spaces are based on $\mathbb{C}$, unless otherwise stated. For convenience, the degree of $x$ or $\varphi$ is denoted by $|x|$ or $|\varphi|$. In addition, $x$ is always assumed to be homogeneous when $|x|$ occurs. Let $L$ be a Lie superalgebra, and denote by $h g(L)$ the set of all homogeneous elements of $L$.

Lie superalgebras as generalizations of Lie algebras were originated from super symmetry in mathematical physics. The theory of Lie superalgebras plays a prominent role in modern mathematics and physics. In recent years, structures of all kinds of Lie superalgebras have aroused many scholars' great interests [1-4]. In this paper, we shall investigate the structure theory of a class of not-finitely graded Lie superalgebras related to the generalized super-Virasoro algebras (namely, derivations, automorphisms, 2-cocycles).

Recently, some researchers have studied structures of some Lie algebras related to the Virasoro algebra [5-10]. The (generalized) superVirasoro algebra is closely related to the conformal field theory and the string theory. It plays a very important role in mathematics and physics. The structure and representation theories of (generalized) superVirasoro algebra have been extensively undertaken by many authors [1,3,11-17]. The generalized super-Virasoro algebra $\operatorname{SVir}[\Gamma, s]$ is a Lie superalgebra whose even part $S V_{\overline{0}}$ has a basis $\left\{L_{\alpha}, C \mid \alpha \in \Gamma\right\}$ and odd part $S V_{\overline{1}}$ has a basis $\left\{G_{\mu} \mid \mu \in \Gamma+s\right\}$, equipped with the following Lie superbrackets:

$$
\begin{aligned}
& {\left[L_{\alpha}, L_{\beta}\right]=(\beta-\alpha) L_{\alpha+\beta}+\frac{\alpha^{3}-\alpha}{12} \delta_{\alpha+\beta, 0} C,} \\
& {\left[L_{\alpha}, G_{\mu}\right]=\left(\mu-\frac{\alpha}{2}\right) G_{\alpha+\mu},} \\
& {\left[G_{\mu}, G_{v}\right]=2 L_{\mu+v}+\frac{1}{3}\left(\mu^{2}-\frac{1}{4}\right) \delta_{\mu+\nu, 0} C,} \\
& {[S V, C]=0,}
\end{aligned}
$$

Where $\alpha, \beta \in \Gamma, \mu, v \in \in s+\Gamma$. If $\Gamma=\mathbb{Z}$, then $\operatorname{SVir}[\mathbb{Z}, s]\left(s=0\right.$ or $\left.\frac{1}{2}\right)$ is the

super-Virasoro algebra. Obviously, $\operatorname{SVir}[\Gamma, s]$ is a generalization of the super-Virasoro algebra. Its theories contain the results of the classical super-Virasoro algebra.

In the present work, we will consider the following Lie superalgebra, called not-finitely graded generalized super-Virasoro algebra $\mathcal{S V}[\Gamma$, $s]$, which has a basis $\left\{L_{\alpha, i}, G_{\mu, j} \mid \alpha \in \Gamma, \mu \in s+\Gamma, i, j \in \mathbb{Z}+\right\}$, and satisfies the following relations:

$$
\begin{aligned}
& {\left[L_{\alpha, i}, L_{\beta, j}\right]=(\beta-\alpha) L_{\alpha+\beta, i+j}+(j-i) L_{\alpha+\beta, i+j-1},} \\
& {\left[L_{\alpha, i}, G_{\mu, j}\right]=\left(\mu-\frac{\alpha}{2}\right) G_{\alpha+\mu, i+j}+\left(j-\frac{i}{2}\right) G_{\alpha+\mu, i+j-1},} \\
& {\left[G_{\mu, i}, G_{v, j}\right]=2 L_{\mu+v, i+j},}
\end{aligned}
$$

Where $\alpha, \beta \in \Gamma, \mu, v \in \mathcal{s}+\Gamma, i, \quad j \in \mathbb{Z}$. We simply denote $\mathcal{S} \mathcal{V}=\mathcal{S V}[\Gamma$, s]. It is obvious that the center of $\mathcal{S V}$ is trival. The Lie algebra $W$ with basis $\left\{L_{\alpha, i} \mid \alpha \in \Gamma, i \in \mathbb{Z}+\right\}$ and relations (5), is the generalized Witt algebra $W=W(0,1,0 ; \Gamma)$ of Witt type studied in [9]. Furthermore, the superalgebra $\mathcal{S} \mathcal{V}$ contains the centerless generalized super-Virasoro algebra as its subalgebra.

If an undefined notation appears in an expression, we treat it as zero. For example, $L_{\alpha}=0, G_{\mu}=0$ if $\alpha \notin \Gamma, \mu \notin s+\Gamma$. The Lie superalgebra $\mathcal{S V}$ is $\Omega$-graded

$$
\begin{aligned}
& \mathcal{S V}=-5 p t_{\alpha \in \Omega}^{\oplus} \mathcal{S} \mathcal{V}_{\alpha}, \mathcal{S} \mathcal{V}_{\alpha}=\operatorname{span}\left\{L_{\alpha, i}, G_{\alpha, i} \mid i \in \mathbb{Z}_{+}\right\} \text {for } \alpha \in \Omega . \\
& \text { Obviously, } \mathcal{S} \mathcal{V}=\mathcal{S} \mathcal{V}_{\overline{0}} \oplus \mathcal{S} \mathcal{V}_{\overline{1}} \text { is } \mathbb{Z}_{2} \text {-graded, where } \\
& \mathcal{S} \mathcal{V}_{\overline{0}}=\operatorname{span}\left\{L_{\alpha, i} \mid \alpha \in \Omega, i \in \mathbb{Z}_{+}\right\}, \mathcal{S} \mathcal{V}_{1}=\operatorname{span}\left\{G_{\mu, i} \mid \mu \in \Omega, i \in \mathbb{Z}_{+}\right\} .
\end{aligned}
$$

Because $\Omega$ may not be finitely generated (as a group), $\mathcal{S V}$ may not be finitely generated as a Lie superalgebra. Hence, some classical techniques [18] cannot be directly applied to this case. We must use some new techniques in order to deal with problems associated with not-finitely generated Lie superalgebras. In addition, in [5,19] some authors studied some structures of not-finitely graded Lie algebras. Thus, some methods about those Lie algebras can be applied in this case. However, it seems to us that little has been known about not-finitely graded aspect of Lie superalgebras. It may be useful and meaningful for promoting the development of not-finitely graded Lie superalgebras. This is the main motivation of this article.

The present paper is organized as follows. In Section 2, we review the basic notions about Lie superalgebras. In Section 3 and Section

*Corresponding author: Guangzhe Fan, Department of Mathematics, Tongji University, Shanghai 200092, PR China, Tel: +73952405-513; E-mail: yzfanguangzhe@126.com

Received September 21, 2016; Accepted November 14, 2016; Published December 14, 2016

Citation: Li J, Fan G (2016) Structures of Not-finitely Graded Lie Superalgebras. J Generalized Lie Theory Appl 10: 247. doi:10.4172/1736-4337.1000247

Copyright: @ $2016 \mathrm{Li} \mathrm{J}$, et al. This is an open-access article distributed under the terms of the Creative Commons Attribution License, which permits unrestricted use, distribution, and reproduction in any medium, provided the original author and source are credited. 
Citation: Li J, Fan G (2016) Structures of Not-finitely Graded Lie Superalgebras. J Generalized Lie Theory Appl 10: 247. doi:10.4172/17364337.1000247

Page 2 of 5

4, we determine the derivations and automorphism groups of $\mathcal{S} \mathcal{V}$, respectively. Finally, the second cohomology groups of $\mathcal{S V}$ are obtained in Section 5. The main results of this paper are summarized in Theorems $3.6,4.2$ and 5.3 .

\section{Preliminaries}

In this section, we shall summarize some basic concepts about Lie superalgebras in $[11,20]$.

\section{Definition 2.1}

A Lie superalgebra is a superalgebra $L=L_{\overline{0}} \oplus L_{\overline{1}}$ with multiplication $[\cdot, \cdot]$ satisfying the following two axioms:

skew super-symmetry: $[x, y]=-(-1)^{|x| y \mid}[y, x]$, super Jacobi identity: $[x,[y, z]]=[[x, y], z]+(-1)^{|x| y}[y,[x, z]]$,

for any $x, y \in h g(L), z \in L$.

Let $L$ be a Lie superalgebra, then the space $g c(L)$ consisting of all the linear transformations on $L$ has a natural $\mathbb{Z}_{2}$-gradation: $g c(L)=g c(L)_{\overline{0}} \oplus g c(L)_{\overline{1}}$, where $g c(L)_{\alpha}=\left\{f \in g c(L) \mid f\left(L_{\beta}\right) \subseteq L_{\alpha+\beta}\right.$, for any $\left.\beta \in \mathbb{Z}_{2}\right\}$, for any $\alpha \in \mathbb{Z}_{2}$. Now we give the definition of derivations.

\section{Definition 2.2}

A derivation of homogenous $\in \mathbb{Z}_{2}$ of $L$ is an $\mathbb{C}$-linear transformation $D \in g c(L)_{\alpha}$ such that

$D([x, y])=[D(x), y]+(-1)^{|D| x \mid}[x, D(y)]$,

for any $x, y \in h g(L)$.

Denote by Der ${ }_{\alpha} L$ the set of all derivations of homogenous $\alpha$ of $L$. Then denote by $\operatorname{Der} L=\operatorname{Der}_{\overline{0}} L \oplus \operatorname{Der}_{\overline{1}} L$ the derivation algebra of $L$. A derivation $\operatorname{D\in Der}(L)$ is called inner if there exists an element $z \in L$ such that $D=a d_{z}$, where $a d_{z}$ is a linear map of $L$ sending $y$ to $[z, y]$ for any $y \in L$. Denote by $a d L$ the set of all inner derivations of $L$.

\section{Definition 2.3}

A bilinear form $\psi: L \times L \rightarrow \mathbb{C}$ is called a 2-cocycle on $L$ if $\psi$ satisfies the following conditions:

skew super-symmetry: $\psi(x, y)=-(-1)^{|x| y \mid} \psi(x, y)$,

super Jacobi identity: $\psi(x,[y, z])=\psi(x, y], z)+(-1)|x||y| \psi(y,[x, z])$,

for any $x, y \in h g(L), z \in L$.

\section{Definition 2.4}

For any linear map $f: L \rightarrow \mathbb{C}$, we define a 2 -cocycle $\psi_{f}$ in the following way:

$\psi f(x, y)=f([x, y])$,

for any $x, y \in L$. We call it a 2-coboundary of $L$.

Denote by $C^{2}(L, \mathbb{C}), B^{2}(L, \mathbb{C})$ the vector spaces of all 2-cocycles, 2-coboundaries of $L$ respectively. The quotient space $H^{2}(L, \mathbb{C})=C^{2}(L, \mathbb{C}) /$ $B^{2}(L, \mathbb{C})$ is called the 2-cohomology group of $L$.

\section{Derivations of $\mathcal{S} \mathcal{V}$}

In this section, we shall determine the derivations of $\mathcal{S V}$.

Denote by $\operatorname{Hom} \mathbb{Z}(\Omega, \mathbb{C})$ the space of group homomorphisms from $\Omega$ to $\mathbb{C}$. For each $\varphi \in \operatorname{Hom} \mathbb{Z}(\Omega, \mathbb{C})$, we define $(c \varphi)(\gamma)=c \varphi(\gamma)$ for any $r \in \Omega, c \in \mathbb{C}$. Furthermore, we define a derivation $D_{\varphi}$ as follows:

$$
D_{\varphi}\left(L_{\alpha, i}\right)=(\alpha) L_{\alpha, i} D_{\varphi}\left(G_{\alpha, i}\right)=\varphi(\alpha) G_{\alpha, i} \text { for } \alpha \in \Omega, \in \mathbb{Z}_{+} .
$$

Denote by $\operatorname{Hom} \mathbb{Z}(\Omega, \mathbb{C})$ the corresponding subspace of Der $\mathcal{S} \mathcal{V}$. In particular, since $\varphi_{0}: \alpha \rightarrow \alpha$ is in $\operatorname{Hom} \mathbb{Z}(\Omega, \mathbb{C})$, we obtain this special derivation

$$
D_{0}=D_{\phi_{0}}: L_{\alpha, i} \mapsto \alpha L_{\alpha, i}, G_{\alpha, i} \mapsto \alpha G_{\alpha, i} \text { for } \alpha \in \Omega, i \in \mathbb{Z}_{+} .
$$

\section{Lemma 3.1}

For every $D \in \mathcal{S V}$, we assume

$$
D=\sum_{\gamma \in \Omega} D_{\gamma}, D_{\gamma} \in(\operatorname{Der} \mathcal{S} \mathcal{V})_{\gamma}
$$

such that only finitely many $D_{\gamma}(x) \neq 0$ for every $x \in \mathcal{S V}$ (such a sum in (11) is called summable).

Proof: For every $D \in \operatorname{Der} \mathcal{S} \mathcal{V}$, we suppose $D\left(x_{\alpha}\right)=\sum_{\beta} \in_{\Omega} y_{\beta}$, where $x_{\alpha} \in \mathcal{S} \mathcal{V}_{\alpha}$. Define $D_{\gamma}\left(x_{\alpha}\right)=y_{\alpha+\gamma}$. Thus, $D_{\gamma}$ is a derivation of $\mathcal{S} \mathcal{V}$.

\section{Lemma 3.2}

For any $D \in \operatorname{Der} \mathcal{S} \mathcal{V}$, replacing $D$ with $D-a d_{\mathrm{y}}$ for some $y \in \mathcal{S} \mathcal{V}$, we get $D\left(L_{0,0}\right)=0$.

Proof: Assume $D\left(L_{0,0}\right)=\sum_{\alpha, j}\left(a_{\alpha, j}^{1} L_{\alpha, j}+a_{\alpha, j}^{2} G_{\alpha, j}\right) \in \mathcal{S V}$ for $a_{\alpha, j}^{\Delta} \in \mathbb{C}$, where $\Delta=1,2$. For any $\alpha \in \Omega$, we define $b_{\alpha, j}^{1}, b_{\alpha, j}^{2} \in \mathbb{C}$ inductively on $j \geq 0$ by

$$
b_{\alpha, j}^{\Delta}= \begin{cases}j^{-1}\left(-a_{\alpha, j-1}^{\Delta}-\alpha b_{\alpha, j-1}^{\Delta}\right), & \text { if } j \geq 1, \\ 0, & \text { if } j=0,\end{cases}
$$

where $\Delta=1,2$.

Choosing $y=\sum_{\alpha, j}\left(b_{\alpha, j}^{1} L_{\alpha, j}+b_{\alpha, j}^{2} G_{\alpha, j}\right) \in \mathcal{S V}$, it follows that

$D\left(L_{0,0}\right)-a d_{y}\left(L_{0,0}\right)=0$.

\section{Lemma 3.3}

If $\gamma \in \Omega^{*}, D \in(\operatorname{Der} \mathcal{S} \mathcal{V})_{\gamma}$ and $D\left(L_{0,0}\right)=0$, then $D=0$.

Proof: Applying $D$ to $\left[L_{0,0}, L_{\alpha, 0}\right]=\alpha L_{\alpha, 0}$ with $\alpha \in \Omega^{*}$, then we get

$$
\left[L_{0,0}, D\left(L_{\alpha, 0}\right)\right]=\alpha D\left(L_{\alpha, 0}\right) \text {, }
$$

Suppose $D\left(L_{\alpha, 0}\right)=\sum_{j \in \mathbb{Z}_{+}}\left(c_{j}^{1} L_{\alpha+\gamma, j}+c_{j}^{2} G_{\alpha+\gamma, j}\right)$ for $c_{j}^{1}, c_{j}^{2} \in \mathbb{C}$, then we obtain

$$
\gamma c_{j}^{\Delta}=-(j+1) c_{j+1}^{\Delta},
$$

for any $j \geq 0$, where $\Delta=1,2$. Thus, $D\left(L_{\alpha, 0}\right)=0$ for any $\alpha \in \Omega$.

Applying $D$ to $\left[L_{0,0}, L_{0,1}\right]=L_{0,0}$, then we obtain

$\left[L_{0,0}, L_{0,1}\right]=D\left(L_{0,0}\right)=0$

Suppose that $D\left(L_{0,1}\right)=\sum_{j \in \mathbb{Z}_{+}}\left(A_{j}^{1} L_{r, j}+A_{j}^{2} G_{r, j}\right)$, where $A_{j}^{1}, A_{j}^{2} \in \mathbb{C}$, then $r A_{j}^{\Delta}=-(j+1) A_{j+1}^{\Delta}$ for any $j \geq 0$, where $\Delta=1,2$. Hence, $D\left(L_{0,1}\right)=0$. Now applying $D$ to $\left[L_{0,0}, G_{\alpha, 0}\right]=\alpha G_{\alpha, 0}$, then it shows

$\left[L_{0,0}, D\left(G_{\alpha, 0}\right)\right]=\alpha D\left(G_{\alpha, 0}\right)$.

Similar to compute $D\left(L_{\alpha, 0}\right)$, we deduce that $D\left(G_{\alpha, 0}\right)=0$ for any $\alpha \in \Omega$.

Since $\mathcal{S V}$ can be generated by $\left\{L_{\alpha, 0}, L_{0,1}, G_{\alpha, 0} \mid \alpha \in \Omega\right\}$, it forces at once that $D=0$.

\section{Lemma 3.4}

Suppose $D \in\left(\operatorname{Der}_{\overline{0}} \mathcal{S V}\right)_{0}, D\left(L_{0,0}\right)=0$, then $D\left(L_{\alpha, 0}\right)=d_{\alpha} L_{\alpha, 0}, D\left(G_{\alpha, 0}\right)=d_{\alpha} G_{\alpha, 0}$ where $d_{\alpha} \operatorname{Hom} \mathbb{Z}(\Omega, \mathbb{C}), \alpha \in \Omega$. 
Citation: Li J, Fan G (2016) Structures of Not-finitely Graded Lie Superalgebras. J Generalized Lie Theory Appl 10: 247. doi:10.4172/17364337.1000247

Proof: Now for any $D \in\left(\text { Der } r_{0} \mathcal{S V}\right)_{0}$, we assume $D\left(L_{\alpha, 0}\right)=\sum_{\alpha, j} d_{j, j} L_{\alpha, j}$, $D\left(G_{\alpha, 0}\right)=\sum_{\alpha, j} e_{\alpha, j} G_{j, j}$ where $d_{\alpha, j} e_{\alpha, j} \in \mathbb{C}$. Applying $D$ to $\left[L_{0,0} L_{\alpha, 0}\right]=\alpha L_{\alpha, 0}$ and $\left[L_{0,0}, G_{\alpha, 0}\right]=\alpha G_{\alpha, 0}$, then we have $d_{\alpha, j}=e_{\alpha, j}=0$ for any $j \geq 1$. Simply denote $d_{\alpha, 0}$, $e_{\alpha, 0}$ by $d_{\alpha}, e_{\alpha}$, respectively. Hence, $D\left(L_{\alpha, 0}\right)=d_{\alpha} L_{\alpha, 0}, D\left(G_{\alpha, 0}\right)=e_{\alpha} G_{\alpha, 0}$ for any $\alpha \in \Omega$. By applying $D$ to $\left[L_{\alpha, 0} L_{\beta, 0}\right]=(-\alpha) L_{\alpha+\beta, 0}$, it has

$d_{\alpha}+d_{\beta}=d_{\alpha+\beta}$ for $\alpha \neq \beta$.

Furthermore, we obtain that the map $\Phi: \alpha \mapsto d_{\alpha}$ is an element in $\operatorname{Hom} \mathbb{Z}(\Omega, \mathbb{C})$. Therefore, $d_{\alpha} \in \operatorname{Hom} \mathbb{Z}(\Omega, \mathbb{C})$.

By using the following three equations

$\left.\left\{\left[L_{\alpha, 0}, G_{\beta, 0}\right]=\left(\beta-\frac{\alpha}{2}\right) G_{\alpha+\beta, 0}, G_{\alpha, 0}, L_{\beta, 0}\right]=\left(\frac{\beta}{2}-\alpha\right) G_{\alpha+\beta, 0}, G_{\alpha, 0}, G_{\beta, 0}\right]=2 L_{\alpha+\beta, 0}$,

we can conclude that

$\left\{\begin{array}{l}d_{\alpha}+e_{\beta}=e_{\alpha+\beta} \text { for } \alpha \neq 2 \beta, \\ e_{\alpha}+d_{\beta}=e_{\alpha+\beta} \text { for } \beta \neq 2 \alpha, \\ e_{\alpha}+e_{\beta}=d_{\alpha+\beta} .\end{array}\right.$

Then, we get $e_{\alpha}=d_{\alpha}$ for any $\alpha \in \Omega$.

Thus, $D\left(L_{\alpha, 0}\right)=d_{\alpha} L_{\alpha, 0}, D\left(G_{\alpha, 0}\right)=d_{\alpha} G_{\alpha, 0}$ for any $\alpha \in \Omega$.

\section{Lemma 3.5}

$$
\begin{aligned}
& \text { If } D\left(L_{0,0}\right)=0 \text {, then } \\
& (\operatorname{Der} \mathcal{S} \mathcal{V})_{0}=(a d \mathcal{S V})_{0} \oplus \operatorname{Hom} \mathbb{Z}(\Omega, \mathbb{C}) .
\end{aligned}
$$

Proof: For any $D \in\left(\operatorname{Der}_{\overline{0}} \mathcal{S V}\right)_{0}$, replacing $D^{\prime}$ by $D-D_{\varphi}$ (cf. (3.1)), then we assume $d_{\alpha}=0$ for any $\alpha \in \Omega$, thus we get $D^{\prime}\left(L_{\alpha, 0}\right)=D^{\prime}\left(G_{\alpha, 0}\right)=0$.

Now assume $D^{\prime}\left(L_{\alpha, i}\right)=\sum_{j \in \mathbb{Z}_{+}} f_{\alpha, j}^{i} L_{\alpha, j}$, where $f_{\alpha, j}^{i} \in \mathbb{C}, i \in \mathbb{Z}_{+}$ . Applying $D^{\prime}$ to $\left[L_{0,0} L_{\alpha, 1}\right]=\alpha L_{\alpha, 1}+L_{\alpha, 0}$, we see $f_{\alpha, j}^{1}=0$ for any $j \geq 1$. Thus, $D^{\prime}\left(L_{\alpha, 1}\right)=f_{\alpha, 0}^{1} L_{\alpha, 0}$ for some $f_{\alpha, 0}^{1} \in \mathbb{C}$. Similarly, applying $D^{\prime}$ to $\left[L_{0,0}, L_{\alpha, 2}\right]=\alpha L_{\alpha, 2}+2 L_{\alpha, 1}$, we obtain

$D^{\prime}\left(L_{\alpha, 2}\right)=f_{\alpha, 0}^{2} L_{\alpha, 0}+2 f_{\alpha, 0}^{1} L_{\alpha, 1}$.

In view of $\left[L_{0,1}, L_{\alpha, 1}\right]=\alpha L_{\alpha, 2}$, it follows

$$
\left\{\begin{array}{l}
\alpha f_{0,0}^{1}=\alpha f_{\alpha, 0}^{1}, \\
f_{0,0}^{1}-f_{\alpha, 0}^{1}=\alpha f_{\alpha, 0}^{2} .
\end{array}\right.
$$

Thus, $f_{\alpha, 0}^{1}=f_{0,0}^{1}, f_{\alpha, 0}^{2}=0$ for $\alpha \neq 0$. Furthermore, according to $\left[L_{-\alpha, 1}\right.$, $\left.L_{\alpha, 1}\right]=2 L_{0,2}$, we get $f_{0,0}^{2}=0$. Hence,

$D^{\prime}\left(L_{\alpha, 1}\right)=f_{0,0}^{1} L_{\alpha, 0}, D^{\prime}\left(L_{\alpha, 2}\right)=2 f_{0,0}^{1} L_{\alpha, 1}$.

Induction on $i$, and use the relations of $W$, then we obtain

$D^{\prime}\left(L_{\alpha, i}\right)=i f_{0,0}^{1} L_{\alpha, i-1}$ for $\alpha \in \Omega, i \in \mathbb{Z}_{+}$.

Applying $D^{\prime}$ to $\left[G_{0,0}, G_{\alpha, i}\right]=2 L_{\alpha, i}$, we have

$D^{\prime}\left(G_{\alpha, i}\right)=i f_{0,0}^{1} G_{\alpha, i}$ for $\alpha \in \Omega, i \in \mathbb{Z}_{+}$.

If we replace $y$ (cf. (3.5)) by $y+f_{0,0}^{1} L_{0,0}$, denote $D^{\prime}+f_{0,0}^{1} D_{0}$ (cf. (3.2)), then $D^{\prime}\left(L_{\alpha, 1}\right)=D^{\prime}\left(G_{\alpha, i}\right)=0$ for $\alpha \in \Omega, i \in \mathbb{Z}_{+}$.

Obviously, $(\operatorname{Der} \mathcal{S V})_{0}=\left(\operatorname{Der}_{\overline{0}} \mathcal{S V}\right)_{0} \oplus\left(\operatorname{Der}_{\overline{1}} \mathcal{S V}\right)_{0}$.

Next for any $D \in\left(\operatorname{Der}_{\mathrm{i}} \mathcal{S V}\right)_{0}$, we suppose $D\left(L_{\alpha, i}\right)=\sum_{j \in \mathbb{Z}_{+}} x_{\alpha, j}^{i} G_{\alpha, j}, D\left(G_{\alpha, i}\right)=\sum_{j \in \mathbb{Z}_{+}} y_{\alpha, j}^{i} L_{\alpha, j}$, where $x_{\alpha, j}^{i}, y_{\alpha, j}^{i} \in \mathbb{C}$. Using the following two equalities, $\left[L_{0,0}, L_{\alpha, 0}\right]=\alpha L_{\alpha, 0}$ and $\left[L_{0,0}, G_{\alpha, 0}\right]=\alpha G_{\alpha, 0}$, then we get $D\left(L_{\alpha, 0}\right)=x_{\alpha} G_{\alpha, 0}, D\left(G_{\alpha, 0}\right)=y_{\alpha} L_{\alpha, 0}$, where $x_{\alpha}=x_{\alpha, 0}^{0}, y_{\alpha}=y_{\alpha, 0}^{0}$. Furthermore, using the relations of $\mathcal{S} \mathcal{V}$, we obtain

$$
D\left(L_{\alpha, i}\right)=D\left(G_{\alpha, i}\right)=0 \text { for any } \alpha \in \Omega, i \in \mathbb{Z}_{+} .
$$

The following theorem can be concluded by Lemmas 3.1-3.5 immediately, which is the main result of this section.

\section{Theorem 3.6}

$\operatorname{Der} \mathcal{S} \mathcal{V}=-5 p t_{\gamma \in \Omega}^{\oplus}(\operatorname{Der} \mathcal{S} \mathcal{V})_{\gamma}=\mathrm{a} d \mathcal{S V} \oplus \operatorname{Hom}_{\mathbb{Z}}(\Omega, \mathbb{C})$,

where $(\operatorname{Der} \mathcal{S} \mathcal{V}) \gamma \subset \mathrm{a} d \mathcal{S} \mathcal{V}$, if $\gamma \in \Omega^{*}$, and $(\operatorname{DerSV}) 0=(\operatorname{adSV}) 0 \oplus \operatorname{Hom} \mathbb{Z}(\Omega, \mathrm{C})$.

\section{Automorphism Groups of $\mathcal{S} \mathcal{V}$} of $\mathcal{S V}$.

The aim of this section is to characterize the automorphism groups

Denote by $\mathrm{A} u t W, \mathrm{~A} u t \mathcal{S} \mathcal{V}$ the automorphism group of $W, \mathcal{S} \mathcal{V}$, respectively. Let $\chi(\Omega)$ be the set of characters of $\Omega$, i.e., the set of group homomorphisms $\tau: \Omega \rightarrow \mathbb{C}^{*}$. Set $\Omega^{\mathbb{C}^{*}}=\left\{c \in \mathbb{C}^{*} \mid c \Omega=\Omega\right\}$. A group structure on $\chi(\Omega) \times \Omega^{\mathbb{C}^{*}}$ can be defined by

$\left(\tau_{1}, c_{1}\right) \cdot\left(\tau_{2}, c_{2}\right)=\left(, c_{1} c_{2}\right)$, where $\tau: \alpha \mapsto \tau_{1}\left(c_{2} \alpha\right) \tau_{2}(\alpha)$ for $\alpha \in \Omega$

Clearly, we kown that $\chi(\Omega) \times \Omega^{\mathbb{C}^{*}}$ is just the semidirect product $\chi(\Omega) \rtimes \Omega^{\mathbb{C}^{*}}$ under the action given by $(c \tau)(\alpha)=\tau(c \alpha)$ for all $c \in \Omega^{\mathbb{C}^{*}}$, $\tau \in \chi(\Omega), \alpha \in \Omega$.

Define a group homomorphism $\varphi:(\tau, c) \mapsto \varphi$ from $\chi(\Gamma) \times \Gamma^{\mathbb{C}^{*}}$ to $\mathrm{A} u t W$ such that $\varphi_{\tau, c}$ is the automorphism of $W$ by

$$
\varphi_{\tau, c}: L_{\alpha, i} \mapsto \tau(\alpha) c^{i-1} L_{c \alpha, i}
$$

where $\alpha \in \Gamma, i \in \mathbb{Z}$. We also define a group homomorphism $\varphi: \tau, \mathcal{C} \mapsto \varphi$ from $\chi(\Omega) \times \Omega^{\mathbb{C}^{*}}$ to AutSV such that $\varphi_{\tau, c}$ is the automorphism of $\mathcal{S} \mathcal{V}$ $\mathcal{S} \mathcal{V}$ by

$$
\begin{aligned}
& \varphi_{\tau, c}: L_{\alpha, i} \mapsto \tau(\alpha) c^{i-1} L_{c \alpha, i}, \\
& \phi_{\tau, c}: G_{\alpha, i} \mapsto \tau(\alpha) c^{i-\frac{1}{2}} G_{c \alpha, i},
\end{aligned}
$$

where $\alpha \in \Omega, i \in \mathbb{Z}_{+}$.

According to [5], we get the following lemma.

\section{Lemma 4.1}

$$
\mathrm{A} u t W \cong \chi(\Gamma) \times \Gamma^{\mathbb{C}^{*}} .
$$

\section{Theorem 4.2}

$$
\mathrm{A} u t \mathcal{S} \mathcal{V} \cong \chi(\Omega) \times \Omega^{\mathbb{C}^{*}} \times \mathbb{Z}_{2} .
$$

Proof: Assume $\sigma \in \mathrm{A} u t \mathcal{S} \mathcal{V}, x \in \mathcal{S} \mathcal{V}_{\overline{0}}$, we assume $\sigma(x)=y+z$, where $y \in \mathcal{S} \mathcal{V}_{\overline{0}}, \quad z \in \mathcal{S} \mathcal{V}_{\overline{1}} .([x, x])=[\sigma(x), \sigma(x)]=[z, z]=0$, then $z=0$. Thus, we have $\sigma\left(L_{\alpha, i}\right)=(\alpha) c^{i-1} L_{c \alpha, i}$ for any $\alpha \in \Omega, i \in \mathbb{Z}_{+}$.

Obviously, $\quad \sigma\left(L_{0,0}\right)=c^{-1} L_{0,0}$. Assume $\quad \sigma\left(G_{\alpha, i}\right)=\Sigma_{\beta, j}\left(A_{\beta, j}^{\alpha, i} L_{\beta, j}+B_{\beta, j}^{\alpha, j} G_{\beta, j}\right)$, where $A_{\beta, j}^{\alpha, i}, B_{\beta, j}^{\alpha, i} \in \mathbb{C}$. Applying $\sigma$ to $\left[L_{0,0}, G_{\alpha, 0}\right]=\alpha G_{\alpha, 0}$, then $\left[c^{-1} L_{0,0}, \Sigma_{\beta, j}\left(A_{\beta, j}^{\alpha, 0} L_{\beta, j}+B_{\beta, j}^{\alpha, 0} G_{\beta, j}\right)\right]=\alpha \Sigma_{\beta, j}\left(A_{\beta, j}^{\alpha, 0} L_{\beta, j}+B_{\beta, j}^{\alpha, 0} G_{\beta, j}\right)$. Comparing the coefficients of $L_{, j}$ and $G_{\beta, j}$, thus, $\beta=c \alpha, A_{c \alpha, j+1}^{\alpha, 0}=B_{c \alpha, j+1}^{\alpha, 0}=0$ for $j \in \mathbb{Z}_{+}$. $A_{\beta, j}^{\alpha, 0}=B_{\beta, j}^{\alpha, 0}=0$ for $\beta \neq c \alpha j \in \mathbb{Z}_{+}$. Hence, $\sigma\left(G_{\alpha, 0}\right)=A_{\alpha} L_{c \alpha, 0}+B_{\alpha} G_{c \alpha, 0}$, where $A_{\alpha}=A_{c \alpha, 0}^{\alpha, 0}, B_{\alpha}=B_{c \alpha, 0}^{\alpha, 0}$.

Next we apply $\sigma$ to $\left[L_{\alpha, 0}, G_{0,0}\right]=-\frac{1}{2} \alpha G_{\alpha, 0}$, then

$$
\left\{\begin{array}{l}
\alpha A_{\alpha}=2 \alpha \tau(\alpha) A_{0} \\
\alpha B_{\alpha}=\alpha \tau(\alpha) B_{0} .
\end{array}\right.
$$

whence, $A_{\alpha}=2 \tau(\alpha) A_{0}$, for $\alpha \in \Omega^{*}$ and $B_{\alpha}=\tau(\alpha) B_{0}$ for $\alpha \in \Omega$. 
Using the equality $\left[G_{0,0}, G_{\alpha, 0}\right]=2 L_{\alpha, 0}$, we have

$$
\left\{\begin{array}{l}
c \alpha A_{0}^{2}+B_{0}^{2}=c^{-1}, \\
\alpha A_{0} B_{0}=0 .
\end{array}\right.
$$

Thus, $A_{0}=0, B_{0}=\nabla c^{-\frac{1}{2}}$, where $\nabla=1$ or -1 .

Hence, $\sigma\left(G_{\alpha, 0}\right)=\nabla c^{-\frac{1}{2}} \tau(\alpha) G_{c \alpha, 0}$ for $\alpha \in \Omega$.

Applying $\sigma$ to $\left[G_{0,0}, G_{\alpha, i}\right]=2 L_{\alpha, i}$, then $\sigma\left(G_{\alpha, i}\right)=A_{0,0}^{\alpha, i} L_{0,0}+\nabla c^{i-\frac{1}{2}} \tau(\alpha) G_{c \alpha, i}$ for any $\alpha \in \Omega, i \in \mathbb{Z}_{+}$. Thanks to $\left[L_{0,0}, G_{\alpha, i}\right]=\alpha G_{\alpha, i}+i G_{\alpha, i-1}$, then we get $A_{0,0}^{\alpha, i}=0$ for any $\alpha \in \Omega, i \in \mathbb{Z}_{+}$.

Finally, $\sigma\left(L_{\alpha, i}\right)=c^{i-1} \tau(\alpha) L_{c \alpha, i}, \sigma\left(G_{\alpha, i}\right)=\nabla c^{i-\frac{1}{2}} \tau(\alpha) G_{c \alpha, i}$, where $\alpha \in \Omega, i \in \mathbb{Z}_{+}$, $\nabla=1$ or -1 .

We have completed the proof.

\section{Second Cohomology Groups of $\mathcal{S V}$}

Let $\psi \in c^{2}(\mathcal{S V} \mathcal{S V}, \mathbb{C})$, we define a linear function $f: \mathcal{S V} \rightarrow \mathbb{C}$ such that $f\left(L_{\alpha, i}\right)$ and $f\left(G_{\alpha, i}\right)$ are given inductively on $i$ as follows

$$
\begin{gathered}
f\left(L_{\alpha, i}\right)= \begin{cases}\frac{1}{i+1} \psi\left(L_{0,0}, L_{0, i+1}\right), & \text { if } \alpha=0, \\
\frac{1}{\alpha}\left(\psi\left(L_{0,0}, L_{\alpha, i}\right)-i f\left(L_{\alpha, i-1}\right)\right), & \text { if } \alpha \neq 0 .\end{cases} \\
f\left(G_{\alpha, i}\right)= \begin{cases}\frac{2}{2 i-1} \psi\left(L_{0,1}, G_{0, i}\right), & \text { if } \alpha=0, \\
\frac{1}{\alpha}\left(\psi\left(L_{0,0}, G_{\alpha, i}\right)-i f\left(G_{\alpha, i-1}\right)\right), & \text { if } \quad \alpha \neq 0 .\end{cases}
\end{gathered}
$$

Set $\varphi=\psi-\psi_{f}$. According to [9], we obtain the following formula:

$\varphi\left(L_{\alpha, i} L_{\beta, j}\right)=0$,

for any $\alpha, \beta \in \Omega, i, j \in \mathbb{Z}_{+}$.

\section{Proposition 5.1}

We have

$\varphi\left(L_{\alpha, i}, G_{\beta, j}\right)=0$,

for any $\alpha, \beta \in \Omega, i, j \in \mathbb{Z}_{+}$.

Proof: Firstly, we have

$\varphi\left(L_{0,0}, G_{\beta, j}\right)=\psi\left(L_{0,0}, G_{j, j}\right)-f\left(\left[L_{0,0}, G_{\beta, j}\right]\right)=0$ for $\beta \neq 0$

One has

$$
\begin{aligned}
& \varphi\left(L_{0,0}, G_{0,0}\right)=2 \varphi\left(L_{0,0},\left[L_{0,1}, G_{0,0}\right]\right) \\
= & 2\left(\varphi\left(L_{0,0}, G_{0,0}\right)+\left(L_{0,1}, 0\right)\right) \\
= & 2\left(\varphi\left(L_{0,0}, G_{0,0}\right) .\right.
\end{aligned}
$$

It is clear that $\varphi\left(L_{0,0}, G_{0,0}\right)$.

Furthermore, for any $j \geq 1$, we obtain

$$
\begin{aligned}
& \varphi\left(L_{0,0}, G_{0, j}\right)=\psi\left(L_{0,0}, G_{0, j}\right)-j f\left(G_{0, j}-1\right) \\
& =\psi\left(L_{0,0}, G_{0, j}\right)-\frac{2 j}{2 j-3} \psi\left(L_{0,1}, G_{0, j-1}\right) \\
& =\psi\left(L_{0,0}, G_{0, j}\right)-\frac{2}{2 j-3} \psi\left(L_{0,1},\left[L_{0,0}, G_{0, j}\right]\right) \\
& =\psi\left(L_{0,0}, G_{0, j}\right)-\frac{2}{2 j-3}\left(-\psi\left(L_{0,0}, G_{0, j}\right)+\frac{2 j-1}{2} \psi\left(L_{0,0}, G_{0, j}\right)\right) \\
& =0 .
\end{aligned}
$$

Thus,

$\varphi\left(L_{0,0}, G_{\beta, j}\right)=0$ for $\beta \in \Omega, j \in \mathbb{Z}_{+}$.

For $\alpha+\beta \neq 0$, it shows

$$
\begin{aligned}
& \phi\left(L_{\alpha, 0}, G_{\beta, 0}\right)=\psi\left(L_{\alpha, 0}, G_{\beta, 0}\right)-\left(\beta-\frac{\alpha}{2}\right) f\left(G_{\alpha+\beta, 0}\right) \\
& =\psi\left(L_{\alpha, 0}, G_{\beta, 0}\right)-\frac{2 \beta-\alpha}{2(\alpha+\beta)} \psi\left(L_{0,0}, G_{\alpha+\beta, 0}\right) \\
& =\psi\left(L_{\alpha, 0}, G_{\beta, 0}\right)-\frac{1}{\alpha+\beta} \psi\left(L_{0,0},\left[L_{\alpha, 0}, G_{\beta, 0}\right]\right)
\end{aligned}
$$

$=0$.

By induction on $i+j$ for $\alpha+\beta \neq 0$, suppose $\varphi\left(L_{\alpha, i-1}, G_{\beta, j}\right)=\varphi\left(L_{\alpha, i}, G_{\beta, j-1}\right)=0$. Applying the Jacobi identity on $\left(L_{0,0}, L_{\alpha, i}, G_{\beta, j}\right)$, we have

$$
\begin{aligned}
& =\varphi\left(L_{0,0},\left[L_{\alpha, i}, G_{\beta, j}\right]\right) \\
= & \varphi\left(\left[L_{0,0}, L_{\alpha, i}\right], G_{\beta, j}\right)+\left(L_{\alpha, i}\left[L_{0,0}, G_{\beta, j}\right]\right) \\
= & (\alpha+\beta) \varphi\left(L_{\alpha, i}, G_{\beta, j}\right) .
\end{aligned}
$$

Hence, we suppose

$$
\varphi\left(L_{\alpha, i}, G_{\beta, j}\right)=\delta_{\alpha+\beta, 0} \varphi\left(L_{\alpha, i}, G_{-\alpha, j}\right) \text { for } \alpha, \beta \in \Omega, i, j \in \mathbb{Z}_{+} .
$$

Next we shall compute $\varphi\left(L_{\alpha, i}, G_{-\alpha, j}\right)$. It follows that

$$
\begin{aligned}
& 0=\varphi\left(L_{0,0},\left[L_{\alpha, 0}, G_{-\alpha, j}\right]\right) \\
& =\varphi\left(\left[L_{0,0}, L_{\alpha, 0}\right], G_{-\alpha, j}\right)+\left(\left[G_{-\alpha, j} L_{0,0}\right], L_{, 0}\right) \\
& =\alpha \varphi\left(L_{\alpha, 0}, G_{-\alpha, j}\right)+\alpha \varphi\left(\left[L_{\alpha, 0}, G_{-\alpha, j}\right], j \varphi\left(L_{\alpha, 0}, G_{-\alpha, j-1}\right)\right. \\
& =j \varphi\left(L_{\alpha, 0}, G_{-\alpha, j-1}\right)
\end{aligned}
$$

Thus, $\varphi\left(L_{\alpha, 0}, G_{-\alpha, j}\right)=0$ for $\alpha \in \Omega, j \in \mathbb{Z}_{+}$.

Induction on $i$, suppose $\varphi\left(L_{\alpha, i-1}, G_{-\alpha, j}\right)=0$, then

$$
\begin{aligned}
& 0=\varphi\left(\left[L_{0,0},\left[L_{\alpha, i}, G_{-\alpha, j}\right]\right)\right. \\
& =\varphi\left(\left[L_{0,0}, L_{\alpha, i}\right], G_{-\alpha, j}\right)+\varphi\left(\left[G_{-\alpha, j}, L_{0,0}\right], L_{\alpha, i}\right) \\
& =\alpha \varphi\left(L_{\alpha, i}, G_{-\alpha, i}\right)+i \varphi\left(\left[L_{\alpha, i-1}, G_{-\alpha, j}\right]-\alpha \varphi\left(L_{\alpha, 0}, G_{-\alpha, j}\right)+j \varphi\left(L_{\alpha, i}, G_{-\alpha, j-1}\right)\right. \\
& =j \varphi\left(L_{\alpha, i}, G_{-\alpha, j-1}\right) .
\end{aligned}
$$

Thus, $\varphi\left(L_{\alpha, i-1}, G_{-\alpha, j}\right)=0$ for any $i, j \in \mathbb{Z}_{+}, \alpha \in \Omega$. This implies that $\varphi($ $\left.L_{\alpha, i} G_{\beta, j}\right)=0$ for $\alpha, \beta \in \Omega, j \in \mathbb{Z}_{+}$.

\section{Proposition 5.2}

We have

$$
\varphi\left(G_{\alpha, i}, G_{\beta, j}\right)=0
$$

for any $\alpha, \beta \in \Omega, i, j \in \mathbb{Z}_{+}$.

Proof: According to the equation (5.3), we deduce that $\varphi\left(L_{0,0}, L_{\alpha, i}\right)=0$ for any $\alpha \in \Omega, i \in \mathbb{Z}_{+}$.

We also obtain that

$$
\begin{aligned}
& 0=\varphi\left(L_{0,0}, 2 L_{\alpha+\beta, 0}\right) \\
& =\varphi\left(L_{0,0},\left[G_{\alpha, 0}, G_{\beta, 0}\right]\right) \\
& =(\alpha+\beta) \varphi\left(G_{\alpha, 0}, G_{\beta, 0}\right) .
\end{aligned}
$$

It turns out that $\varphi\left(G_{\alpha, 0}, G_{\beta, 0}\right)=0$ for $\alpha+\beta \neq 0$. Induction on $i+j$, suppose $\varphi\left(G_{\alpha, i-1}, G_{\beta, j}\right)=\varphi\left(G_{\alpha, i}, G_{\beta, j-1}\right)=0$. Then we get

$$
0=\varphi\left(L_{0,0}, 2 L_{\alpha+\beta, i+j}\right)
$$




$$
\begin{aligned}
& =\varphi\left(L_{0,0},\left[G_{\alpha, i}, G_{\beta, j}\right]\right) \\
& =(\alpha+\beta) \varphi\left(G_{\alpha, i} G_{\beta, j}\right)+j \varphi\left(G_{\alpha, i} G_{\beta, j-1}\right)+i \varphi\left(G_{\alpha, i-1}, G_{\beta, j}\right) \\
& =(\alpha+\beta) \varphi\left(G_{\alpha, i}, G_{\beta, j}\right) .
\end{aligned}
$$

Therefore, $\varphi\left(G_{\alpha, j} G_{\beta, j}\right)=0$ for $\alpha+\beta \neq 0$. So in the following, we shall only be concerned with the case $\alpha+\beta \neq 0$.

$$
\begin{aligned}
& \text { If } j \in \mathbb{Z}_{+}, \text {then } \\
& \phi\left(G_{0,0}, G_{0, j}\right)=\frac{1}{j+1} \phi\left(G_{0,0},\left[L_{0,0}, G_{0, j+1}\right]\right) \\
& =\frac{1}{j+1}\left(\phi\left(\left[G_{0,0}, L_{0,0}\right], G_{0, j+1}\right)+\phi\left(L_{0,0},\left[G_{0,0}, G_{0, j+1}\right]\right)\right) \\
& =\frac{1}{j+1} \phi\left(L_{0,0}, 2 L_{0, j+1}\right) . \\
& =0 .
\end{aligned}
$$

Induction on $i$, suppose $\varphi\left(G_{0, i-1}, G_{0, j}\right)=0$ for any $j \in \mathbb{Z}_{+}$, then

$$
\begin{aligned}
& 0=\varphi\left(L_{0,0}, 2 L_{0, i+j}\right) \\
& =\varphi\left(L_{0,0},\left[G_{0, i} G_{0, j}\right]\right) \\
& =\varphi\left(\left[L_{0,0}, G_{0, i}\right], G_{0, j}\right)+\varphi\left(G_{0, i}\left[L_{0,0}, G_{0, j}\right]\right) \\
& =i \varphi\left(G_{0, i-1}, G_{0, j}\right)+j \varphi\left(G_{0, i} G_{0, j-1}\right) \\
& =j \varphi\left(G_{0, i}, G_{0, j-1}\right) .
\end{aligned}
$$

Thus, $\varphi\left(G_{0, i}, G_{0, j}\right)=0$ for any $i, j \in \mathbb{Z}_{+}$. Furthermore, it follows that

$$
\begin{aligned}
& 0=\varphi\left(L_{0,0}, 2 L_{0, i}\right) \\
= & \varphi\left(L_{0,0},\left[G_{\alpha, i}, G_{-\alpha, 0}\right]\right) \\
= & i \varphi\left(G_{\alpha, i-1}, G_{-\alpha, j}\right) .
\end{aligned}
$$

Hence, $\left(G_{\alpha, i}, G_{-\alpha, 0}\right)=0$ for any $i \in \mathbb{Z}_{+}$.

Applying the Jacobi identity on $\left(L_{0,0}, G_{\alpha, i}, G_{-\alpha, j}\right)$, then we get

$$
0=2 \varphi\left(L_{0,0}, L_{0, i+j}\right)
$$$$
=\varphi\left(L_{0,0}, G_{\alpha, i}, G_{-\alpha, j}\right)
$$$$
=i \varphi\left(G_{\alpha, i-1}, G_{-\alpha, j}\right)+j \varphi\left(G_{\alpha, i}, G_{-\alpha, j-1}\right) .
$$

Induction on $j$, we also obtain $\left(G_{\alpha, i} G_{-\alpha, j}\right)=0$

By previous computations, it will suffice to show the result.

Now we get the main result of this section.

\section{Theorem 5.3}

The second cohomology group of $\mathcal{S V}$ is trivial, i.e.

$\mathrm{C}^{2}(\mathcal{S V}, \mathbb{C})=\mathrm{B}^{2}(\mathcal{S V}, \mathbb{C})$

\section{Conclusion}

From the above study one can conlcude that Lie superalgebras as generalizations of Lie algebras were originated from supersymmetry in mathematical physics. The theory of Lie superalgebras plays a prominent role in modern mathematics and physics. The (generalized) super-Virasoro algebra is closely related to the conformal field theory and the string theory. It plays a very important role in mathematics and physics. The paper is devoted in the investigation of the structure theory of class of not finitely graded Lie superalgebras related to generalized super-Virasoro algebras. In particular, the derivation algebras, the automorphism groups and the second cohomology groups of these Lie algebras are determined.

\section{References}

1. Dai X, Han J, Su Y (2015) Structures of generalized loop Super-Virasoro algebras. Internat J Math 26: 1550041.

2. Hoyt C (2012) Good gradings of basic Lie superalgebras. Israel J Math 192 251-280.

3. lohara K, Koga $\mathrm{Y}$ (2004) Representation theory of $\mathrm{N}=2$ super Virasoro algebra twisted sector. J Funct Anal 214: 450-518.

4. Wang $Y$, Pei $Y$, Deng S (2014) Leibniz central extensions of Lie superalgebras J Algebra Appl 13: 1450052.

5. Chen Q, Han J, Su Y (2015) Structures of not finitely graded Lie algebras related to generalized Virasoro algebras. Comm Algebra 43: 3033-3049.

6. Dokovic D, Zhao K (1998) Derivations, isomorphisms, and second cohomology of generalized Witt algebras. Trans Amer Math Soc 350: 643-664.

7. Patera J, Zassenhaus H (1991) The higher rank Virasoro algebras. Comm Math Phys 136: 1-14.

8. Su Y, Xu X, Zhang H (2000) Derivation-simple algebras and the structures of Lie algebras of Witt type. J Algebra 233: 642-662.

9. Su Y, Zhao K (2002) Second cohomology group of generalized Witt type Lie algebras and certain representations. Comm Algebra 30: 3285-3309.

10. Wu H, Wang S, Yue X (2014) Structures of generalized loop Virasoro algebras Comm Algebra 42: 1545-1558.

11. Sato HT (1997) Deformation of super Virasoro algebra in non-commutative quantum super-Space. Phys Lett B 415: 170-174.

12. Scheunert M (1979) The Theory of Lie Superalgebras. Lecture Notes in Mathematics 716 .

13. Su Y (1994) Harish-Chandra modules of intermediate series over the high rank Virasoro algebras and high rank super-Virasoro algebras. J Math Phys 35 2013-2023.

14. Su Y (1995) Classification of Harish-Chandra modules over the super-Virasoro algebras. Comm Algebra 23: 3653-3675.

15. Su Y, Zhao K (2002) Generalized Virasoro and super-Virasoro algebras and modules of the intermediate series. J Algebra 252: 1-19.

16. Yang H (2009) Lie super-bialgebra structures on super-Virasoro algebras Front Math China 4: 365-379.

17. Zhang $X$ (2015) Classification of simple weight modules for super-Virasoro algebra with finite-dimensional weight space. Front Math China 10: 1233-1242.

18. Farnsteiner $R$ (1988) Derivations and central extensions of finitely generated graded Lie algebras. J Algebra 118: 33-45.

19. Fan G, Zhou C, Yue X (2015) Structures of not-_nitely graded Lie algebras related to generalized Heisenberg-Virasoro algebras. Acta Math Sin 31: 15171530.

20. Kac VG (1977) Lie superalgebras. Adv Math 26: 8-96.

Citation: Li J, Fan G (2016) Structures of Not-finitely Graded Lie Superalgebras. J Generalized Lie Theory Appl 10: 247. doi:10.4172/1736-4337.1000247 